\title{
LOWER BOUND FOR THE REGULARITY INDEX OF FAT POINTS
}

\author{
Phan Van Thien \\ Department of Mathematics \\ Hue Normal University \\ VIETNAM
}

\begin{abstract}
The problem to find an upper bound for the regularity index of fat points has been dealt with by many authors. In this paper we give a lower bound for the regularity index of fat points. It shall be an useful tool for determining the regularity index.
\end{abstract}

AMS Subject Classification: 14C20, 13D40

Key Words: regularity index, fat points

\section{Introduction}

Let $P_{1}, \ldots, P_{s}$ be distinct points in the projective space $\mathbb{P}^{n}:=\mathbb{P}^{n}(k), k$ an algebraically closed field. Denote by $\wp_{1}, \ldots, \wp_{s}$ the prime ideals in the polynomial ring $R:=k\left[X_{0}, \ldots, X_{n}\right]$ corresponding to the points $P_{1}, \ldots, P_{s}$. Let $m_{1}, \ldots, m_{s}$ be positive integers. We will denote by $Z$ the zero-scheme defined by the ideal $I:=\wp_{1}^{m_{1}} \cap \cdots \cap \wp_{s}^{m_{s}}$ and call $Z$ a set of fat points in $\mathbb{P}^{n}$.

The homogeneous coordinate ring of $Z$ is $R / I$. This ring is a one-dimensional Cohen-Macaulay graded ring, $R / I=\underset{t \geq 0}{\oplus}(R / I)_{t}$, whose multiplicity is

$$
e(R / I)=\sum_{i=1}^{s}\left(\begin{array}{c}
m_{i}+n-1 \\
n
\end{array}\right) .
$$

The function $H_{R / I}(t):=\operatorname{dim}_{k}(R / I)_{t}$ strictly increases until it reaches the mul-

Received:

Revised:

Published: September 30, 2016 (c) 2016 Academic Publications, Ltd. url: www.acadpubl.eu 
tiplicity $e(R / I)$, at which it stabilizes. The regularity index of $Z$, denote by $\operatorname{reg}(Z)$, is defined to be the least integer $t$ such that $H_{R / I}(t)=e(R / I)$. It is well known that $\operatorname{reg}(Z)=\operatorname{reg}(R / I)$, the Castelnuovo-Mumford regularity of $R / I$. Hence we will also denote $\operatorname{reg}(Z)$ by $\operatorname{reg}(R / I)$.

The problem to exactly determine the regularity index $\operatorname{reg}(Z)$ is fairly difficult. So, instead of determining $\operatorname{reg}(Z)$, one tries to find an upper bound for it. The problem to find an upper bound for $\operatorname{reg}(Z)$ has been dealt with by many authors (see [1]-[14]). In this paper we will give a lower bound for the regularity index of fat points. The lower bound and upper bound are useful tools for determining the regularity index.

The algebraic method used in this paper as well as in [6], [12], [13], [14].

\section{Preliminaries}

From now on, we say a $j$-plane, i.e. a linear $j$-space. We will identify a hyperplane as the linear form defining it.

We will use the following lemmas which have been proved in [6].

Lemma 1. [6, Lemma 1] Let $P_{1}, \ldots, P_{r}, P$ be distinct points in $\mathbb{P}^{n}$ and let $\wp$ be the defining ideal of $P$. If $m_{1}, \ldots, m_{r}$ and $a$ are positive integers, $J:=\wp_{1}^{m_{1}} \cap \cdots \cap \wp_{r}^{m_{r}}$, and $I=J \cap \wp^{a}$, then

$$
\operatorname{reg}(R / I)=\max \left\{a-1, \operatorname{reg}(R / J), \operatorname{reg}\left(R /\left(J+\wp^{a}\right)\right)\right\} .
$$

Lemma 2. [6, Lemma 3] Let $P_{1}, \ldots, P_{r}, P$ be distinct points in $\mathbb{P}^{n}$ and let $\wp$ be the defining ideal of $P$. Let $a, m_{1}, \ldots, m_{r}$ positive integers. Put $J=\wp_{1}^{m_{1}} \cap \cdots \cap \wp_{r}^{m_{r}}$ and $\wp=\left(X_{1}, \ldots, X_{n}\right)$. Then

$$
\operatorname{reg}\left(R /\left(J+\wp^{a}\right)\right) \leq b
$$

if and only if $X_{0}^{b-i} M \in J+\wp^{i+1}$ for every monomial $M$ of degree $i$ in $X_{1}, \ldots, X_{n}$, $i=0, \ldots, a-1$.

Suppose that we can find $t$ hyperplanes $H_{1}, \ldots, H_{t}$ avoiding $P$ such that $H_{1} \cdots H_{t} M \in J$ for every monomial $M$ of degree $i$ in $X_{1}, \ldots, X_{n}, i=0, \ldots, a-$ 1. Since we can write $H_{j}=X_{0}+G_{j}$ for some linear form $G_{j} \in \wp$ for $j=1, \ldots, t$, we get $X_{0}^{t} M \in J+\wp^{i+1}$. Therefore, we have the following lemma:

Lemma 3. Assume that $H_{1}, \ldots, H_{t}$ are hyperplanes avoiding $P$ such that $H_{1} \cdots H_{t} M \in J$ for every monomial $M$ of degree $i$ in $X_{1}, \ldots, X_{n}, i=$ $0, \ldots, a-1$. If

$$
\delta \geq \max \{t+i \mid 0 \leq i \leq a-1\}
$$


then

$$
\operatorname{reg}\left(R /\left(J+\wp^{a}\right)\right) \leq \delta
$$

The following lemma has been proved in [14].

Lemma 4. $\left[14\right.$, Lemma 3.3] Let $X=\left\{P_{1}, \ldots, P_{s}\right\}$ be a set of distinct points in $\mathbb{P}^{n}$, and $m_{1}, \ldots, m_{s}$ be positive integers. Put $I=\wp_{1}^{m_{1}} \cap \cdots \cap \wp_{s}^{m_{s}}$. If $Y=\left\{P_{i_{1}}, \ldots, P_{i_{r}}\right\}$ is a subset of $X$ and $J=\wp_{i_{1}}^{m_{i_{1}}} \cap \cdots \cap \wp_{i_{r}}^{m_{i_{r}}}$, then

$$
\operatorname{reg}(R / I) \geq \operatorname{reg}(R / J)
$$

\section{Lower Bound for the Regularity Index of Fat Points}

Let $X=\left\{P_{1}, \ldots, P_{s}\right\}$ be a set of distinct points in $\mathbb{P}^{n}$ and $m_{1}, \ldots, m_{s}$ be positive integers. Let $n_{1}, \ldots, n_{s}$ be non-negative integers with $\left(n_{1}, \ldots, n_{s}\right) \neq(0, \ldots, 0)$ and $m_{i} \geq n_{i}$ for $i=1, \ldots, s$. Put $I=\wp_{1}^{m_{1}} \cap \cdots \cap \wp_{s}^{m_{s}}, N=\wp_{1}^{n_{1}} \cap \cdots \cap \wp_{s}^{n_{s}}$ $\left(\wp_{i}^{n_{i}}=R\right.$ if $\left.n_{i}=0\right)$. Then we have $e(R / I) \geq e(R / N)$ and $H_{R / I}(t) \geq H_{R / N}(t)$. So, we can not compare $\operatorname{reg}(R / I)$ with $\operatorname{reg}(R / N)$ by definition of the regularity index. In Proposition 6 we will prove that $\operatorname{reg}(R / I) \geq \operatorname{reg}(R / N)$.

The first, we get the following result.

Lemma 5. Let $X=\left\{P_{1}, \ldots, P_{s}\right\}$ be a set of distinct points in $\mathbb{P}^{n}$ and $m_{1}, \ldots, m_{s}, n_{1}, \ldots, n_{s}$ be positive integers with $m_{i} \geq n_{i}$ for $i=1, \ldots, s$. Put $I=\wp_{1}^{m_{1}} \cap \cdots \cap \wp_{s}^{m_{s}}$ and $N=\wp_{1}^{n_{1}} \cap \cdots \cap \wp_{s}^{n_{s}}$, then

$$
\operatorname{reg}(R / I) \geq \operatorname{reg}(R / N)
$$

Proof. In case $m_{i}=n_{i}$ for $i=1, \ldots, s$, we have the equality. In case there exists $j$ such that $m_{j}>n_{j}$, we may assume that $m_{s}>n_{s}$. Put $I_{1}=$ $\wp_{1}^{m_{1}} \cap \cdots \cap \wp_{s-1}^{m_{s-1}} \cap \wp_{s}^{m_{s}-1}$. We will prove $\operatorname{reg}(R / I) \geq \operatorname{reg}\left(R / I_{1}\right)$.

Put $J=\wp_{1}^{m_{1}} \cap \cdots \cap \wp_{s-1}^{m_{s-1}}$. By Lemma 2 we have

$$
\begin{aligned}
& \operatorname{reg}\left(R /\left(J+\wp_{s}^{m_{s}}\right)\right) \leq b \\
& \Leftrightarrow X_{0}^{b-i} M \in J+\wp_{s}^{i+1} \text { for every } M=X_{1}^{c_{1}} \cdots X_{n}^{c_{n}}, c_{1}+\cdots+c_{n}=i, \\
& \quad i=0, \ldots, m_{s}-1 \\
& \Rightarrow X_{0}^{b-i} M \in J+\wp_{s}^{i+1} \text { for every } M=X_{1}^{c_{1}} \cdots X_{n}^{c_{n}}, c_{1}+\cdots+c_{n}=i, \\
& \quad i=0, \ldots, m_{s}-2 \\
& \Leftrightarrow \operatorname{reg}\left(R /\left(J+\wp_{s}^{m_{s}-1}\right)\right) \leq b .
\end{aligned}
$$


This implies $\operatorname{reg}\left(R /\left(J+\wp_{s}^{m_{s}}\right)\right) \geq \operatorname{reg}\left(R /\left(J+\wp_{s}^{m_{s}-1}\right)\right)$. By Lemma 1 we have

$$
\begin{aligned}
\operatorname{reg}(R / I) & =\max \left\{m_{s}-1, \operatorname{reg}(R / J), \operatorname{reg}\left(R /\left(J+\wp_{s}^{m_{s}}\right)\right)\right\} . \\
\operatorname{reg}\left(R / I_{1}\right) & =\max \left\{m_{s}-2, \operatorname{reg}(R / J), \operatorname{reg}\left(R /\left(J+\wp_{s}^{m_{s}-1}\right)\right)\right\} .
\end{aligned}
$$

Therefore, we get

$$
\operatorname{reg}(R / I) \geq \operatorname{reg}\left(R / I_{1}\right)
$$

By inductive argue on $m_{s}$ we get

$$
\operatorname{reg}(R / I) \geq \operatorname{reg}\left(R /\left(\wp_{1}^{m_{1}} \cap \cdots \cap \wp_{s-1}^{m_{s-1}} \cap \wp_{s}^{n_{s}}\right)\right)
$$

By induction on number of points we get

$$
\operatorname{reg}(R / I) \geq \operatorname{reg}(R / N)
$$

From the above lemma and Lemma 4 we get the following proposition.

Proposition 6. Let $X=\left\{P_{1}, \ldots, P_{s}\right\}$ be a set of distinct points in $\mathbb{P}^{n}$ and $m_{1}, \ldots, m_{s}$ be positive integers. Let $n_{1}, \ldots, n_{s}$ be non-negative integers with $\left(n_{1}, \ldots, n_{s}\right) \neq(0, \ldots, 0)$ and $m_{i} \geq n_{i}$ for $i=1, \ldots, s$. Put $I=\wp_{1}^{m_{1}} \cap \cdots \cap \wp_{s}^{m_{s}}$, $N=\wp_{1}^{n_{1}} \cap \cdots \cap \wp_{s}^{n_{s}}\left(\wp_{i}^{n_{i}}=R\right.$ if $\left.n_{i}=0\right)$. We have

$$
\operatorname{reg}(R / I) \geq \operatorname{reg}(R / N)
$$

A rational normal curve in $\mathbb{P}^{j}$ to be a curve of degree $j$ that may be given parametrically as the image of the map

$$
\begin{aligned}
\mathbb{P}^{1} & \rightarrow \mathbb{P}^{j} \\
(s, t) & \mapsto\left(s^{n}, s^{j-1} t, s^{j-2} t^{2}, \ldots, t^{j}\right) .
\end{aligned}
$$

Let $Q_{1}, \ldots, Q_{r}$ be distinct points on a linear $j$-space, say $\alpha$, in $\mathbb{P}^{n}$. If there exist a rational normal curve, say $\mathcal{C}$, in $\mathbb{P}^{j}$ and an isomorphism of a linear change of coordinates

$$
\varphi: \mathbb{P}^{j} \rightarrow \alpha
$$

such that $Q_{1}, \ldots, Q_{r} \in \varphi(\mathcal{C})$, then we said that $Q_{1}, \ldots, Q_{r}$ are in Rnc-j. In $\mathbb{P}^{n}$ the points whose coordinators satisfying parametric equations

$$
X_{0}=t^{j}, X_{1}=t^{j-1} u, \ldots, X_{j-1}=t u^{j-1}, X_{j}=u^{j}, X_{j+1}=\cdots=X_{n}=0
$$


lie on a image of a rational normal curve in $\mathbb{P}^{j}$ by an isomorphism of a linear change of coordinates. So, if $Q_{1}, \ldots, Q_{r}$ are in Rnc-j in $\mathbb{P}^{n}$, then we may assume that their coordinators satisfying the above parametric equations.

Let $Z=m_{1} P_{1}+\cdots+m_{s} P_{s}$ be a set of fat points in $\mathbb{P}^{n}$. Then the set

$$
\left\{P_{i_{1}}, \ldots, P_{i_{q}} \in\left\{P_{1}, \ldots, P_{s}\right\} \mid P_{i_{1}}, \ldots, P_{i_{q}} \text { are in } R n c-1\right\}
$$

is non-empty.

The following theorem shows a lower bound for the regularity index of fat points.

Theorem 7. Let $Z=m_{1} P_{1}+\cdots+m_{s} P_{s}$ be a set of fat points in $\mathbb{P}^{n}$. Then,

$$
\operatorname{reg}(Z) \geq \max \left\{D_{j} \mid j=1, \ldots, n\right\}
$$

where

$$
D_{j}=\max \left\{\left[\frac{\sum_{l=1}^{q} m_{i_{l}}+j-2}{j}\right] \mid P_{i_{1}}, \ldots, P_{i_{q}} \text { are in Rnc-j }\right\} .
$$

Proof. Suppose that points $P_{i_{1}}, \ldots, P_{i_{q}}$ of $\left\{P_{1}, \ldots, P_{n}\right\}$ are Rnc-j in $\mathbb{P}^{n}$. We may assume that $m_{i_{1}} \geq \cdots \geq m_{i_{q}}$ (after relabeling the points, if necessary). Let $\wp_{i_{1}}, \ldots, \wp_{i_{q}}$ be the homogeneous prime ideals of $R$ corresponding to the points $P_{i_{1}}, \ldots, P_{i_{q}}$. Put

$$
J=\wp_{1}^{m_{i_{1}}} \cap \cdots \cap \wp_{i_{q}}^{m_{i_{q}}}
$$

By Lemma 4 we have

$$
\operatorname{reg}(Z) \geq \operatorname{reg}(R / J)
$$

We will prove that

$$
\operatorname{reg}(R / J) \geq\left[\frac{\sum_{l=1}^{q} m_{i_{l}}+j-2}{j}\right]
$$

Since the points $P_{i_{1}}, \ldots, P_{i_{q}}$ are in Rnc-j in $\mathbb{P}^{n}$, we may assume that their coordinators satisfying parametric equations:

$$
X_{0}=t^{j}, X_{1}=t^{j-1} u, \ldots, X_{j-1}=t u^{j-1}, X_{j}=u^{j}, X_{j+1}=\cdots=X_{n}=0
$$

and the points $P_{i_{q}}=(1,0, \ldots, 0)$. Then $\wp_{i_{q}}=\left(X_{1}, \ldots, X_{n}\right)$. Put

$$
J_{1}=\wp_{i_{1}}^{m_{i_{1}}} \cap \cdots \cap \wp_{i_{q-1}}^{m_{i_{q-1}}} .
$$


The first, we will prove that

$$
\operatorname{reg}\left(R /\left(J_{1}+\wp_{i_{q}}^{m_{i_{q}}}\right)\right) \geq\left[\frac{\sum_{l=1}^{q} m_{i_{l}}+j-2}{j}\right] .
$$

Put $T=\left[\frac{\sum_{l=1}^{q} m_{i_{l}}+j-2}{j}\right]$. Consider the monomial $X_{1}^{m_{i_{q}}-1}$. If

$$
X_{0}^{T-m_{i_{q}}} X_{1}^{m_{i_{q}}-1} \in J_{1}+\wp_{i_{q}}^{m_{i_{q}}},
$$

then there exists a form $h \in \wp_{i_{q}}^{m_{i_{q}}}$ of degree $T-1$ such that

$$
X_{0}^{T-m_{i_{q}}} X_{1}^{m_{i_{q}}-1}+h \in J_{1} \text {. }
$$

Since $X_{0}^{T-m_{i_{q}}} X_{1}^{m_{i_{q}}-1} \in \wp_{i_{q}}^{m_{i_{q}}-1}$ and $h \in \wp_{i_{q}}^{m_{i_{q}}} \subset \wp_{i_{q}}^{m_{i_{q}}-1}$, we have

$$
X_{0}^{T-m_{i_{q}}} X_{1}^{m_{i_{q}}-1}+h \in J_{1} \cap \wp_{i_{q}}^{m_{i_{q}}-1} \text {. }
$$

Moreover, $m_{i_{1}}+\cdots+m_{i_{q}}-1>j(T-1)$, hence by Bezout's theorem

$$
X_{0}^{T-m_{i_{q}}} X_{1}^{m_{i_{q}}-1}+h
$$

vanishing on the points $\left(1, \lambda, \ldots, \lambda^{j}, 0, \ldots, 0\right) \in \mathbb{P}^{n}$, for every $\lambda$ in the field $k$. This implies

$$
\lambda^{m_{i_{q}}-1}+h\left(1, \lambda, \ldots, \lambda^{j}, 0, \ldots, 0\right)=0
$$

for every $\lambda \in k$. Since $h \in \wp_{i_{q}}^{m_{i_{q}}}=\left(X_{1}, \ldots, X_{n}\right)^{m_{i_{q}}}$, we have $h\left(1, \lambda, \ldots, \lambda^{j}, 0\right.$, $\ldots, 0)=0$ or $h\left(1, \lambda, \ldots, \lambda^{j}, 0, \ldots, 0\right)=\lambda^{m_{i_{q}}} g(\lambda)$, for some non-zero polynomial $g \in k[x]$. Hence, $\lambda^{m_{i_{q}}-1}=0$ or $\lambda^{m_{i_{q}}-1}+\lambda^{m_{i_{q}}} g(\lambda)=0$ for every $\lambda \in k$, a contradiction. Thus, we get

$$
X_{0}^{T-m_{i_{q}}} X_{1}^{m_{i_{q}}-1} \notin J_{1}+\wp_{i_{q}}^{m_{i_{q}}} .
$$

By Lemma 2 we have

$$
\operatorname{reg}\left(R /\left(J_{1}+\wp_{i_{q}}^{m_{i_{q}}}\right)\right) \geq T .
$$

Next, by Lemma 1 we get

$$
\begin{aligned}
\operatorname{reg}(R / J) & =\max \left\{m_{i_{q}}-1, \operatorname{reg}\left(R / J_{1}\right), \operatorname{reg}\left(R /\left(J_{1}+\wp_{i_{q}}^{m_{i_{q}}}\right)\right)\right\} \\
& \geq \operatorname{reg}\left(R /\left(J_{1}+\wp_{i_{q}}^{m_{i_{q}}}\right)\right) \geq T .
\end{aligned}
$$

The proof of Theorem 7 is now completed. 


\section{Application of Lower Bound}

The first, by using the lower bound we can compute the regularity index of fat points whose support on a line. This formula was showed by E.D. Davis and A.V. Geramita in [7] by using another method.

Proposition 8. Let $Z=m_{1} P_{1}+\cdots+m_{s} P_{s}$ be a set of fat points in $\mathbb{P}^{n}$. If $P_{1}, \ldots, P_{s}$ lie on a line, then

$$
\operatorname{reg}(Z)=m_{1}+\cdots+m_{s}-1 .
$$

Proof. We may assume that $m_{1} \geq \cdots \geq m_{s}$. If $P_{1}, \ldots, P_{s}$ lie on a line, then $D_{1}=m_{1}+\cdots+m_{s}-1$. Put $I=\wp_{1}^{m_{1}} \cap \cdots \cap \wp_{s}^{m_{s}}$. We will prove that

$$
\operatorname{reg}(R / I)=D_{1} \text {. }
$$

By Theorem 7 we have

$$
\operatorname{reg}(R / I) \geq \max \left\{D_{j} \mid j=1, \ldots, n\right\} .
$$

So, it suffices to prove by induction on $s$ that

$$
\operatorname{reg}(R / I) \leq D_{1}
$$

Put $J=\wp_{1}^{m_{1}} \cap \cdots \cap \wp_{s-1}^{m_{s-1}}$, by the inductive assumption, we get

$$
\operatorname{reg}(R / J) \leq m_{1}+\cdots+m_{s-1}-1 \leq D_{1}
$$

Choose $P_{s}=(1,0, \ldots, 0)$, then $\wp_{s}=\left(X_{1}, \ldots, X_{n}\right)$. Since $P_{1}, \ldots, P_{s}$ lie on a line, there exists hyperplane, say $H_{j}$, passing throught $P_{j}$ and avoiding $P_{s}$ for $j=1, \ldots, s-1$. This implies

$$
H_{1}^{m_{1}} \cdots H_{s-1}^{m_{s-1}} \in J
$$

Therefore, for every monomial $M=X_{1}^{c_{1}} X_{2}^{c_{2}} \cdots X_{n}^{c_{n}}$ of degree $i, i=0, \ldots, m_{s}-$ 1 , we have

$$
H_{1}^{m_{1}} \cdots H_{s-1}^{m_{s-1}} M \in J
$$

By Lemma 3 we get

$$
\operatorname{reg}\left(R /\left(J+\wp_{s}^{m_{s}}\right)\right) \leq D_{1} .
$$

From (1), (2) and Lemma 1 we get

$$
\operatorname{reg}(R / I) \leq D_{1} .
$$


Now we consider a set of fat points whose support is in Rnc-j.

Lemma 9. Let $Z=m_{1} P_{1}+\cdots+m_{s} P_{s}$ be a set of fat points in $\mathbb{P}^{n}$. Suppose that $j$ is the least integer such that $P_{1}, \ldots, P_{s}$ are in Rnc-j. If $t$ is an integer such that

$$
t \geq \max \left\{m_{l},\left[\frac{m_{1}+\cdots+m_{s-1}+j-1}{j}\right] \mid l=1, \ldots, s-1\right\},
$$

then we can find $t$ hyperplanes, say $H_{1}, \ldots, H_{t}$, avoiding $P_{s}$ sucth that

$$
H_{1} \cdots H_{t} \in \wp_{1}^{m_{1}} \cap \cdots \cap \wp_{s-1}^{m_{s-1}} .
$$

Proof. Since the points $P_{1}, \ldots, P_{s}$ are in Rnc-j in $\mathbb{P}^{n}$, we may assume that their coordinators satisfying parametric equations:

$$
X_{0}=v^{j}, X_{1}=v^{j-1} u, \ldots, X_{j-1}=v u^{j-1}, X_{j}=u^{j}, X_{j+1}=\cdots=X_{n}=0 .
$$

If $t=1$, then $P_{1}, \ldots, P_{s}$ lie on a line. For $j=1, \ldots, s-1$, there exists a hyperplane, say $H_{j}$, passing throught $P_{j}$ and avoiding $P_{s}$. Then we have $t=$ $m_{1}+\cdots+m_{s-1}$ hyperplanes $\underbrace{H_{1}, \ldots, H_{1}}_{m_{1}}, \ldots, \underbrace{H_{s-1}, \ldots, H_{s-1}}_{m_{s-1}}$ avoiding $P_{s}$ such that

$$
H_{1}^{m_{1}} \cdots H_{s-1}^{m_{s-1}} \in \wp_{1}^{m_{1}} \cap \cdots \cap \wp_{s-1}^{m_{s-1}} .
$$

If $t \geq 2$, then no $l+2$ points of $\left\{P_{1}, \ldots, P_{s}\right\}$ are on a $l$-plane for $l<j$. This implies that there does not exist any $(j-1)$-plane containing $j+1$ points of $\left\{P_{1}, \ldots, P_{s}\right\}$. We will prove the lemma by induction on $\sum_{i=1}^{s-1} m_{i}$.

We may assume that $m_{1} \geq \cdots \geq m_{s-1}$. Since $j$ is the least integer such that $P_{1}, \ldots, P_{s}$ are in Rnc-j, we have $j \leq s-1$. Let $\sigma_{1}$ be the $(j-1)$-plane passing throught $P_{1}, \ldots, P_{j}$. Then $\sigma_{1}$ avoids $P_{s}$. Therefore, there is a hyperplane, say $L_{1}$, containing $\sigma_{1}$ and avoiding $P_{s}$.

Case $s-1=j$ : Then

$$
L_{1}^{t} \in \wp_{1}^{m_{1}} \cap \cdots \cap \wp_{s-1}^{m_{1}} \subset \wp_{1}^{m_{1}} \cap \cdots \cap \wp_{s-1}^{m_{s-1}} .
$$

Case $s-1 \geq j+1$ : Since $t \geq\left[\frac{m_{1}+\cdots+m_{s-1}+j-1}{j}\right]$ and $m_{1} \geq \cdots \geq m_{s-1}$, we have

$$
\begin{aligned}
t-1 & \geq\left[\frac{m_{1}+\cdots+m_{s-1}+j-1}{j}\right]-1 \geq\left[\frac{(j+1) m_{j+1}-1}{j}\right] \\
& \geq m_{j+1} .
\end{aligned}
$$


On the other hand, since $t \geq\left[\frac{m_{1}+\cdots+m_{s-1}+j-1}{j}\right]$, we get

$$
t-1 \geq\left[\frac{\left(m_{1}-1\right)+\cdots+\left(m_{j}-1\right)+m_{j+1}+\cdots+m_{s-1}+j-1}{j}\right] .
$$

Consider

$$
Z_{1}=\left(m_{1}-1\right) P_{1}+\cdots+\left(m_{j}-1\right) P_{j}+m_{j+1} P_{j+1}+\cdots+m_{s-1} P_{s-1}+m_{s} P_{s} .
$$

By the inductive assumption we can find $(t-1)$ hyperplanes, say $L_{2}, \ldots, L_{t}$, avoiding $P_{s}$ such that

$$
L_{2} \cdots L_{t} \in \wp_{1}^{m_{1}-1} \cap \cdots \cap \wp_{j}^{m_{j}-1} \cap \wp_{j+1}^{m_{j+1}} \cap \cdots \cap \wp_{s-1}^{m_{s-1}} .
$$

Moreover, since $L_{1} \in \wp_{1} \cap \cdots \cap \wp_{j}$, we get

$$
L_{1} L_{2} \cdots L_{t} \in \wp_{1}^{m_{1}} \cap \cdots \cap \wp_{s-1}^{m_{s-1}} .
$$

We can compute the regularity index of fat points whose support is in Rnc-j.

Proposition 10. Let $Z=m_{1} P_{1}+\cdots+m_{s} P_{s}$ be a set of fat points in $\mathbb{P}^{n}$. If $P_{1}, \ldots, P_{s}$ are in Rnc-t, then

$$
\operatorname{reg}(Z)=\max \left\{D_{j} \mid j=1, \ldots, t\right\}
$$

where

$$
D_{j}=\max \left\{\left[\frac{\sum_{l=1}^{q} m_{i_{l}}+j-2}{j}\right] \mid P_{i_{1}}, \ldots, P_{i_{q}} \text { are in Rnc-j }\right\} .
$$

Proof. We may assume that $m_{1} \geq \cdots \geq m_{s}$. We will argue by induction on $s$. If $s=1$, then $\operatorname{reg}(Z)=m_{1}-1=D_{1}$. If $s \geq 2$, we consider two following cases:

Case $t=1$ : Then $P_{1}, \ldots, P_{s}$ lie on a line and $D_{1}=m_{1}+\cdots+m_{s}-1=$ $\max \left\{D_{j} \mid j=1, \ldots, n\right\}$. By Proposition 8 we have $\operatorname{reg}(Z)=D_{1}$.

Case $t \geq 2$ : Since $P_{1}, \ldots, P_{s}$ are in Rnc-t, there is the least integer $p \leq t$ such that $P_{1}, \ldots, P_{s}$ are in Rnc-p. Then

$$
\begin{aligned}
& D_{1}=m_{1}+m_{2}-1 \geq D_{2} \geq \cdots \geq D_{p-1} \\
& D_{p}=\left[\frac{m_{1}+\cdots+m_{s}+p-2}{p}\right] \geq D_{p+1} \geq \cdots \geq D_{n} .
\end{aligned}
$$


So, $\max \left\{D_{j} \mid j=1, \ldots, n\right\}=\max \left\{D_{j} \mid j=1, \ldots, t\right\}=\max \left\{D_{1}, D_{p}\right\}$. Hence, by Theorem 7 we get

$$
\operatorname{reg}(Z) \geq \max \left\{D_{1}, D_{p}\right\}
$$

It sufficies to prove that

$$
\operatorname{reg}(Z) \leq \max \left\{D_{1}, D_{p}\right\}
$$

Put $Z_{1}=m_{1} P_{1}+\cdots+m_{s-1} P_{s-1}, J=\wp_{1}^{m_{1}} \cap \cdots \cap \wp_{s-1}^{m_{s-1}}$ and $Y=\left\{P_{1}, \ldots, P_{s-1}\right\}$. We have $\operatorname{reg}\left(Z_{1}\right)=\operatorname{reg}(R / J)$. By inductive hypothesis we have

$$
\operatorname{reg}\left(Z_{1}\right)=\max \left\{D_{j}^{\prime} \mid j=1, \ldots, t\right\},
$$

where

$$
D_{j}^{\prime}=\max \left\{\left[\frac{\sum_{l=1}^{q} m_{i_{l}}+j-2}{j}\right] \mid Y \ni P_{i_{1}}, \ldots, P_{i_{q}} \text { are in Rnc-j }\right\} .
$$

Since $\left\{P_{1}, \ldots, P_{s-1}\right\} \subset\left\{P_{1}, \ldots, P_{s-1}, P_{s}\right\}$, we have $D_{j}^{\prime} \leq D_{j}$ for $j=1, \ldots, t$. Therefore, we get

$$
\operatorname{reg}(R / J) \leq \max \left\{D_{1}, D_{p}\right\}
$$

Consider $R /\left(J+\wp_{s}^{m_{s}}\right)$. We may assume that

$$
P_{s}=(1,0, \ldots, 0), P_{1}=(0, \underbrace{1}_{2}, 0, \ldots, 0), \ldots, P_{p}=(0, \ldots, 0, \underbrace{1}_{p+1}, 0, \ldots, 0) .
$$

For every monomial $M=X_{1}^{c_{1}} \cdots X_{n}^{c_{n}}, c_{1}+\cdots+c_{n}=i, i=0, \ldots, m_{s}-1$. Put

$$
m_{l}^{\prime}= \begin{cases}m_{l}-i+c_{l} & \text { for } l=1, \ldots, p \\ m_{l} & \text { for } l=p+1, \ldots, s-1\end{cases}
$$

Put $J^{\prime}=\wp_{1}^{m_{1}^{\prime}} \cap \cdots \cap \wp_{s-1}^{m_{s-1}^{\prime}}$. By Proposition 4 we can find

$$
t=\max \left\{m_{l}^{\prime},\left[\frac{m_{1}^{\prime}+\cdots+m_{s-1}^{\prime}+p-1}{p}\right] \mid l=1, \ldots, s-1\right\}
$$

hyperplanes, say $H_{1}, \ldots, H_{t}$, avoiding $P_{s}$ such that

$$
H_{1} \cdots H_{t} \in J^{\prime}
$$

Since $M \in \wp_{1}^{i-c_{1}} \cap \cdots \cap \wp_{p}^{i-c_{p}}$ and $J^{\prime}=\wp_{1}^{m_{1}-i+c_{1}} \cap \cdots \cap \wp_{p}^{m_{p}-i+c_{p}} \cap \wp_{p+1}^{m_{p+1}} \cdots \cap$ $\wp_{s-1}^{m_{s-1}}$, we get

$$
H_{1} \cdots H_{t} M \in J
$$


By Lemma 3 we get

$$
\operatorname{reg}\left(R /\left(J+\wp_{s}^{m_{s}}\right)\right) \leq \max \left\{t+i \mid i=1, \ldots, m_{s}-1\right\} \leq \max \left\{D_{1}, D_{p}\right\} .
$$

Put $I=J \cap \wp_{s}^{m_{s}}$. We have $\operatorname{reg}(Z)=\operatorname{reg}(R / I)$. From (3), (4) and Lemma 1 we have

$$
\operatorname{reg}(Z) \leq \max \left\{D_{1}, D_{p}\right\}
$$

\section{References}

[1] E. Ballico, On the Segre upper bound of the regularity for fat points in $\mathbb{P}^{4}$, I, IJPAM, Vol 102, No.2, (2015), 281-300.

[2] E. Ballico, On the Segre upper bound of the regularity for fat points in $\mathbb{P}^{4}$, II, IJPAM, Vol 102, No.2, (2015), 301-347.

[3] E. Ballico, On the Segre upper bound of the regularity for non-fat point schemes in projective spaces, IJPAM, Vol 102, No.2, (2015), 273-279.

[4] E. Ballico, O. Dumitrescu, and E. Postinghel, On Segre's Bound for fat points in $P^{n}$, J. Pure and Appl. Algebra 220, Issue 6, (2016), 2307-2323.

[5] B. Benedetti, G. Fatabbi and A. Lorenzini, Segre's Bound and the case of $n+2$ fat points of $P^{n}$, Comm. Algebra 40 (2012), 395-403.

[6] M.V. Catalisano, N.V. Trung and G. Valla, A sharp bound for the regularity index of fat points in general position, Proc. Amer. Math. Soc. 118 (1993), 717-724.

[7] E.D. Davis and A.V. Geramita, The Hilbert function of a special class of 1-dimensional Cohen-Macaulay graded algebras, The Curves Seminar at Queen's, Queen's Papers in Pure and Appl. Math. 67 (1984), 1-29.

[8] G. Fatabbi, Regularity index of fat points in the projective plane, J. Algebra 170 (1994), 916-928.

[9] G. Fatabbi, A. Lorenzini On a sharp bound for the regularity index of any set of fat points, J. Pure and Appl. Algebra 161 (2001), 91-111.

[10] W. Fulton, Algebraic Curves, Math. Lect. Note Series, Benjamin 1969.

[11] B. Segre, Alcune questioni su insiemi finiti di punti in geometria algebrica, Atti. Convergno. Intern. di Torino 1961, 15-33.

[12] P.V. Thien, Segre bound for the regularity index of fat points in $\mathbb{P}^{3}$, J. Pure and Appl. Algebra 151 (2000), 197-214.

[13] P.V. Thien, Sharp upper bound for the regularity of zero-schemes of double points in $\mathbb{P}^{4}$, Comm. Algebra 30 (2002), 5825-5847.

[14] P.V. Thien, Regularity index of $s+2$ fat points not on a linear $(s-1)$-space, Comm. Algebra 40 (2012), 3704-3715. 
